POLLACK PERIODICA

An International Journal for Engineering and Information Sciences

DOI: $10.1556 / 606.2016 .11 .1 .8$

Vol. 11, No. 1, pp. 81-90 (2016)

www.akademiai.com

\title{
TWO NEW SPACE-FILLING MOSAICS BASED ON A SYMMETRIC 3D MODEL OF THE 10D CUBE
}

\author{
László VÖRÖS \\ Department of Architectural and Visual Studies, Faculty of Engineering and Information \\ Technology, University of Pécs, Boszorkány u. 2, H-7624 Pécs, Hungary \\ e-mail: vorosl@pmmik.pte.hu
}

Received 2 February 2015; accepted 28 August 2015

\begin{abstract}
Two new periodical space-filling mosaics will be constructed. These are based on a 3D model of the 10D cube. The initial edges have the same lengths and are placed rotation symmetrically around an axis, thus the model is a specifically arranged zonotope. Each mosaic consists of this model and some derived ones of lower-dimensional parts of the 10D cube. These two tessellations complete the construction series based on similar models of 3-12D cubes except the $11 \mathrm{D}$ one.
\end{abstract}

Keywords: Hypercube, Spatial and planar tessellations

\section{The considered 3D model of a hypercube}

Modeling of a hypercube has more or less known precedents. Some related references are listed at the end of the paper and in [1], [2]. The analogous construction method of the next 3-model is described in more details in [1], [3], [4].

Lifting the vertices of a $k$-sided regular polygon from their plane, perpendicularly to the same height, and joining them with the center of the polygon, can be get the $k$ initial edges of the $k$-dimensional cube (further on $k$-cube) modeled in the three-dimensional space (3-model). From these the 3-models or their polyhedral surface (Fig. 1) can also be generated by the well-known procedure. That is by moving the lower-dimensional elements along edges parallel with the direction of the next dimension [3], [1], [4]. Thus each polyhedron will become a zonotope [1], [5], [6], i.e. a 'translational sum' of some segments (Minkowski-type sum) [3], [4]. 


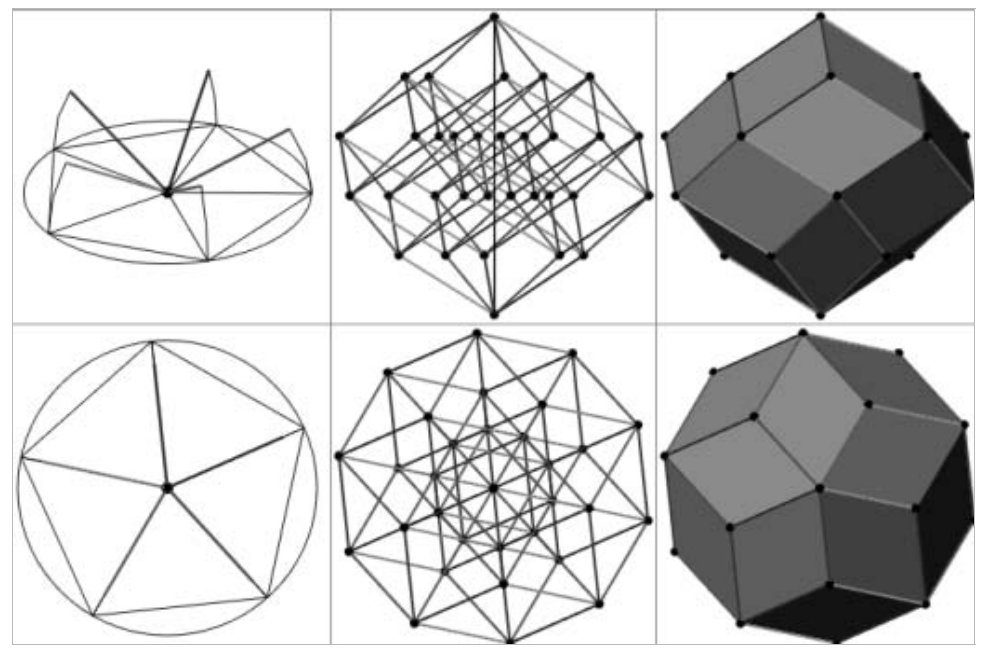

Fig. 1. The considered symmetric 3-model of the 5-cube

This structure keeps the normal cube's central symmetry and rotational symmetry too. The latter is related to the diagonal joining the starting vertex, referred to the collections of any $j<k$ dimensioned elements. This diagonal is called further on as main diagonal (Fig. 1, upper middle). It is also possible to get to the endpoint of the main diagonal from the starting point along easily recognizable strut-chains, whose binding points (the outer vertices of the model) join helices (Fig. 2). The common creator of the cylinder surfaces, joining the helices is the above mentioned main diagonal. The whole 3-model can be constructed as sequences of strut-chains originated from a separate one whose breakpoints join a single helix. Increasing the number of segments in the strutchains to large $k=n$, a real helix can be created, and the Minkowski-sum of the infinitely many helices is called $n$-zonotope. In this way the 3 -model of each $k$-dimensional cube fits into this construction [1].
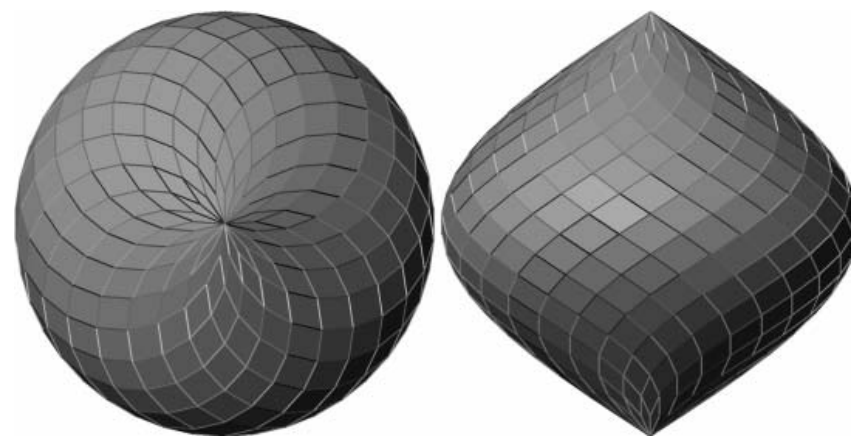

Fig. 2. Top and front views of the 3-model of the 21-cube 


\section{Space-filling periodical tessellations of 3-models of $j \leq k$-cubes}

Combining $2<j<k$ initial edges of the above model, 3-models of $j$-cubes, as parts of a k-cube can be built (Fig. 3). The suitable combinations of these models can result in space-filling mosaics. The presented periodical tessellations (up to $k=12$ ) always hold the 3-model of the $k$-cube and of the necessary $j$-cubes derived from it (Fig. 4).

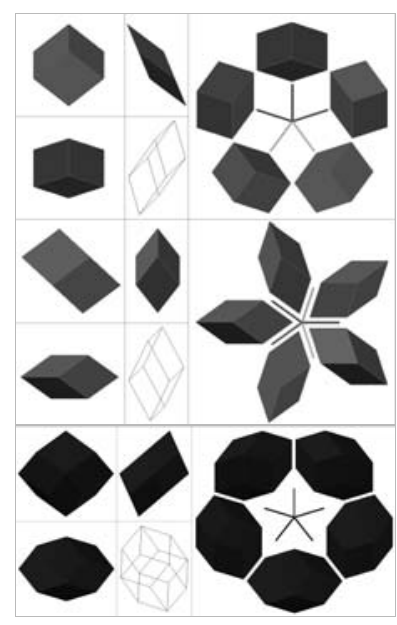

Fig. 3. 3-models of 3- and 4-cubes based on 5 given edges

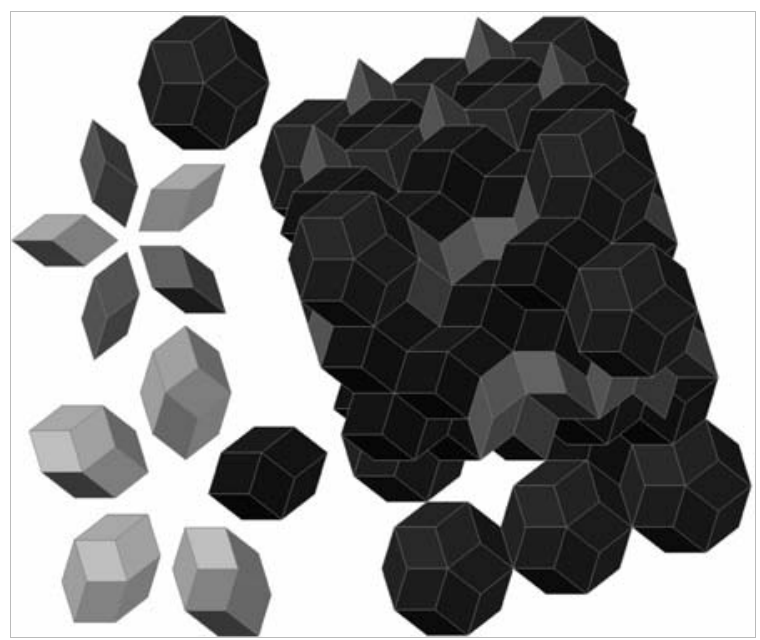

Fig. 4. An example of tessellation based on the 3-model of the 5-cube

The related computer animations [7] show horizontal intersections of similar 3D tessellations, as periodical 2D-mosaic. Varying the vertical height of these intersections the vertical periodicity can be also guaranteed. Finally a so-called parallelohedron is constructed experimentally from the above described elementary polyhedra, whose translated images fill the 3D-space without gaps and overlaps.

\section{Two space-filling periodical tessellations based on the 10 -cube}

As it is exemplified in Fig. 3, there are chosen 3 up to $k$ edges of the rotational symmetric arranged initial ones in different combinations to construct the 3-models of the $j<k$ dimensional parts of the $k$-cube. Table $I$ shows how many differently shaped elements exist, derived from the above described 3-model of the 10-cube.

The presented tessellations are spatial mosaics having two kinds of layers of the 3 -model of the 10-cube and those of the derived lower dimensional elements. One type of the layers consists of the initial model of the 10-cube as well, the other one only of 
the derived models. These stones of the mosaics have horizontal and vertical symmetry planes (Fig. 5, Fig. 6).

Table I

How many kinds of derived elements has the considered 3-model of the 10-cube

\begin{tabular}{|r|c|c|c|c|c|c|c|c|}
\hline dimensions & 3 & 4 & 5 & 6 & 7 & 8 & 9 & 10 \\
amounts of elements & 12 & 22 & 26 & 22 & 12 & 5 & 1 & 1 \\
\hline
\end{tabular}
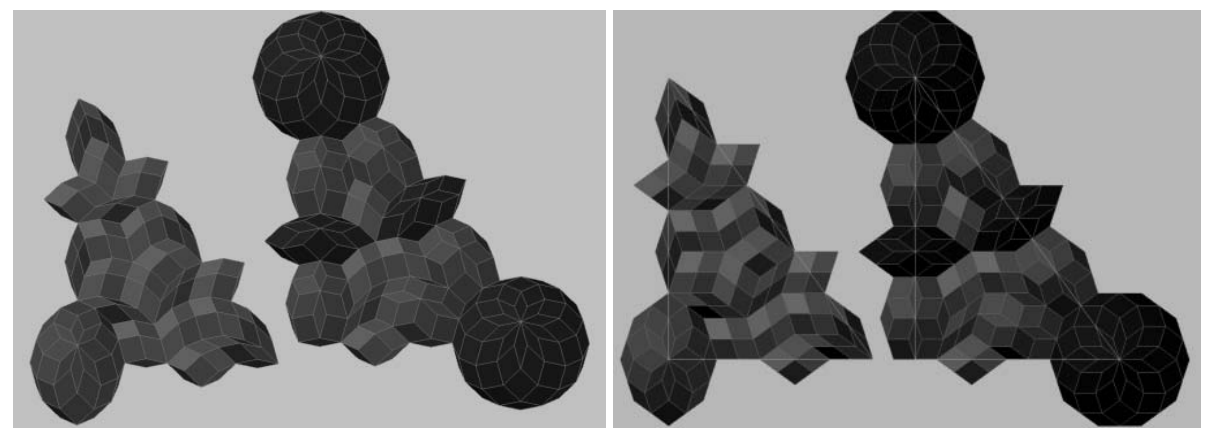

Fig. 5. The unit layers of mosaic 1 in axonometric projection and in top view
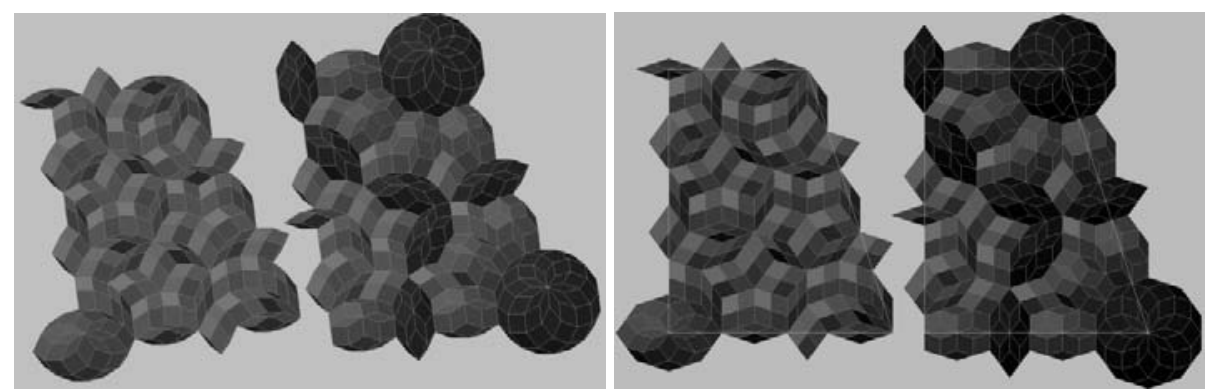

Fig. 6. The unit layers of mosaic 2 in axonometric projection and in top view

The unit triangular and trapezium shaped parts create the secondary rhombic and hexagonal unit parts. The discussed 3-model of the 10-cube has a tenfold rotational symmetry, so the angles between the vertical border planes of the secondary unit parts are two times and three times 36 degrees in mosaic 1 (Fig. 7) and these are three times and four times 36 degrees in mosaic 2 (Fig. 8). The elements along the border lines are common members of the neighboring parts. The above two different layers of the unit parts alternate each other vertically. The space among these is filled by the derived elements also in translated and mirror arrangements.

The layouts of the stones joining vertical border planes of the mosaic units can be seen in Fig. 9 and Fig. 10. The considered planes are perpendicular to each other in between the spatial tessellation. It can be recognized that the arrangement of the 
elements is also mirrored to the horizontal mirror planes of the repeated special layers of stones.
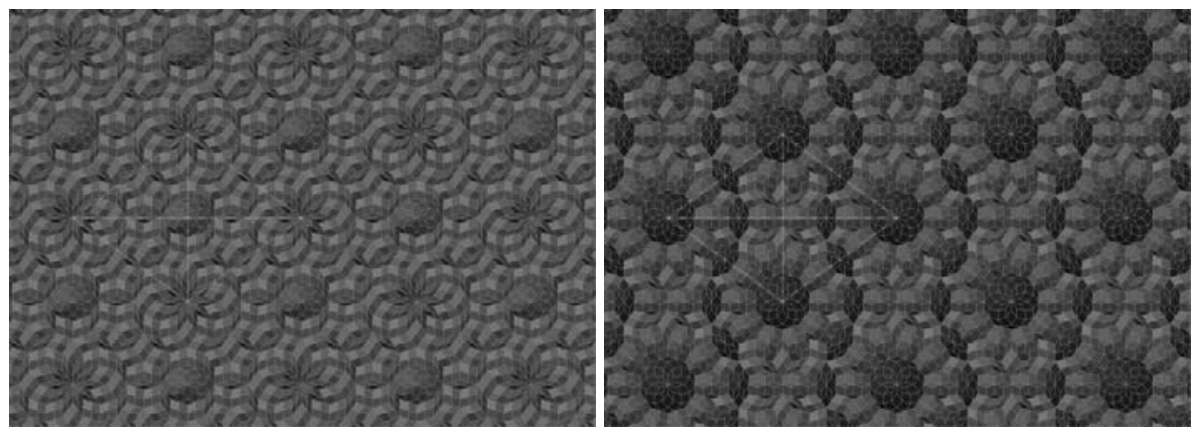

Fig. 7. Mosaic 1, The horizontally symmetric layers 1 and 2 in top view, The vertical border planes of the units are indicated with light lines
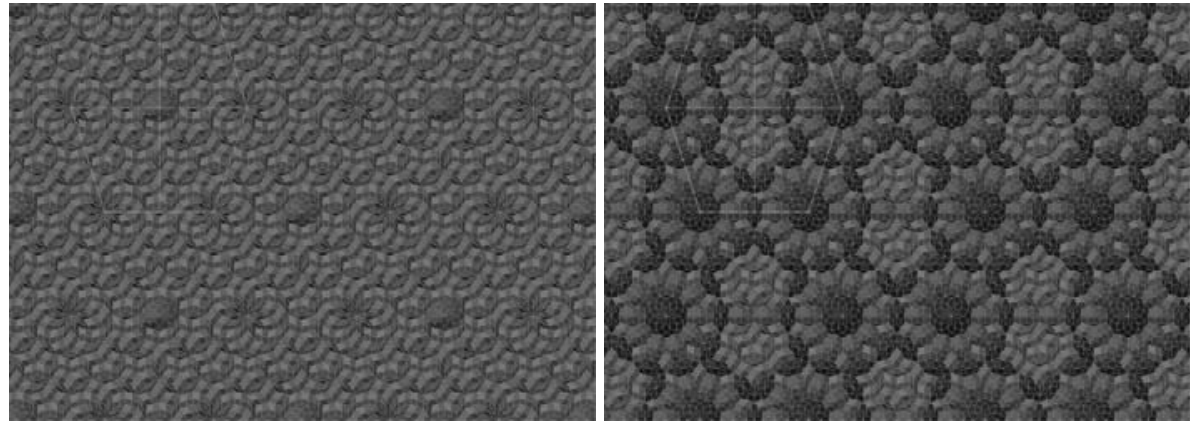

Fig. 8. Mosaic 2, The horizontally symmetric layers 1 and 2 in top view, The vertical border planes of the units are indicated with light lines
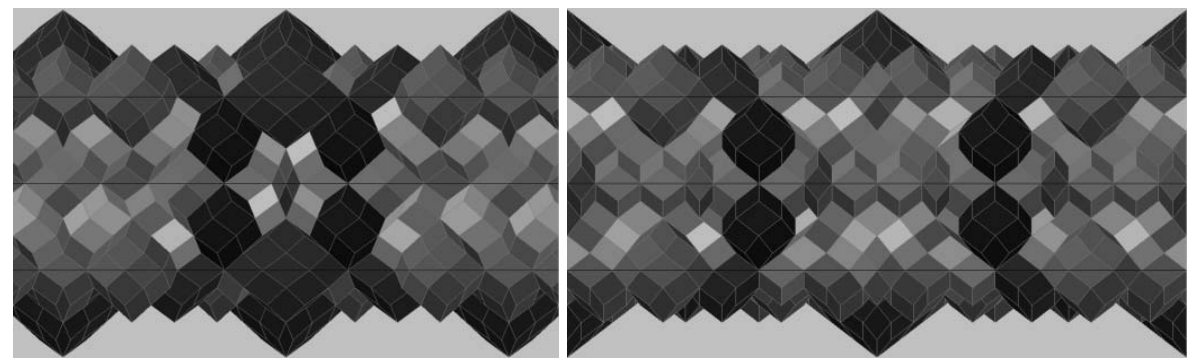

Fig. 9. Mosaic 1, Layouts of stones joining different vertical border planes

The construction of mosaic 1 requires 16 differently shaped stones. Table II shows the distribution of these derived elements according to the numbers of dimensions. In the case of mosaic 2, the construction requires the 16 stones of the mosaic 1 and 2 additional ones. The distribution of the elements shows Table III. 


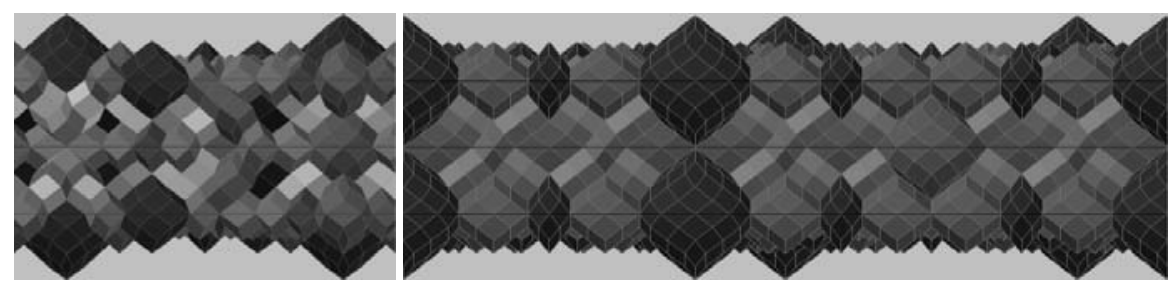

Fig. 10. Mosaic 2, Layouts of stones joining different vertical border planes

Table II

How many kinds of differently shaped elements are used in mosaic 1

\begin{tabular}{|r|c|c|c|c|c|c|c|c|}
\hline dimensions & 3 & 4 & 5 & 6 & 7 & 8 & 9 & 10 \\
amounts of elements & 7 & 4 & 1 & 2 & 0 & 1 & 0 & 1 \\
\hline
\end{tabular}

Table III

How many kinds of differently shaped elements are used in mosaic 2

\begin{tabular}{|r|c|c|c|c|c|c|c|c|}
\hline dimensions & 3 & 4 & 5 & 6 & 7 & 8 & 9 & 10 \\
amounts of elements & 7 & 5 & 2 & 2 & 0 & 1 & 0 & 1 \\
\hline
\end{tabular}

The structure of the second mosaic is a little bit more sophisticated. Fig. 11-Fig. 17 show the distribution of stones with different symmetry properties and numbers of dimensions in the two horizontally symmetric layers as well as in between them in top view. This means that elements placed on different levels can hide each other.

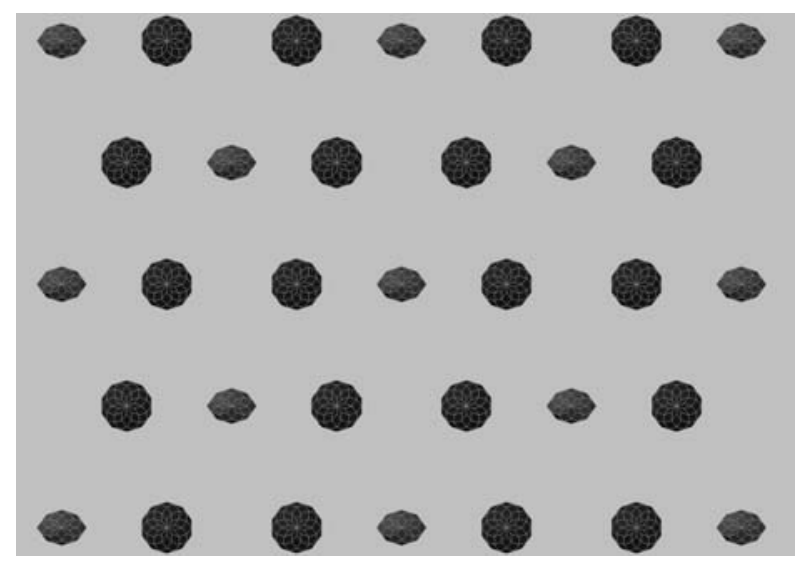

Fig. 11. The used $8 \mathrm{D}$ and $10 \mathrm{D}$ elements with vertical and horizontal symmetry planes 


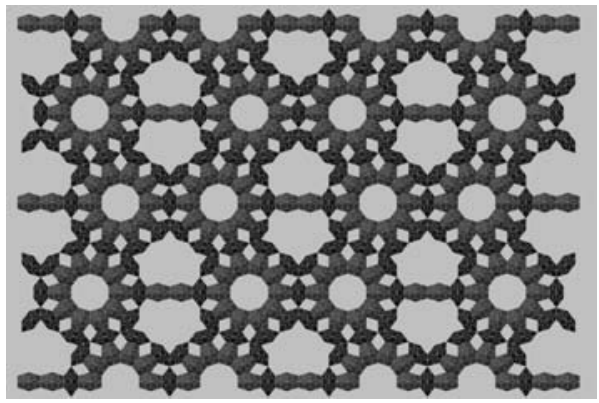

Fig. 12. 2 differently shaped 6D elements with vertical and horizontal symmetry planes

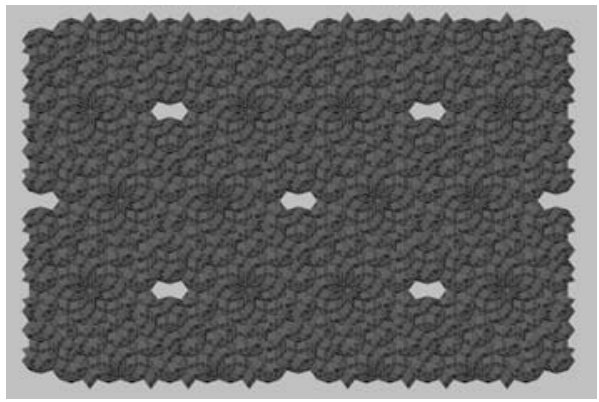

Fig. 14. 2 differently shaped 4D elements with vertical and horizontal symmetry planes

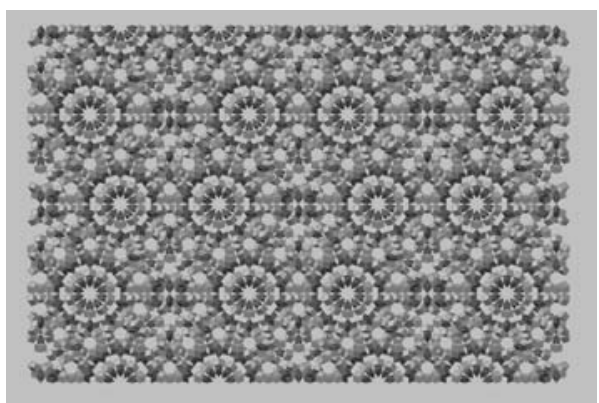

Fig. 16. 3 differently shaped 3D elements with vertical symmetry planes

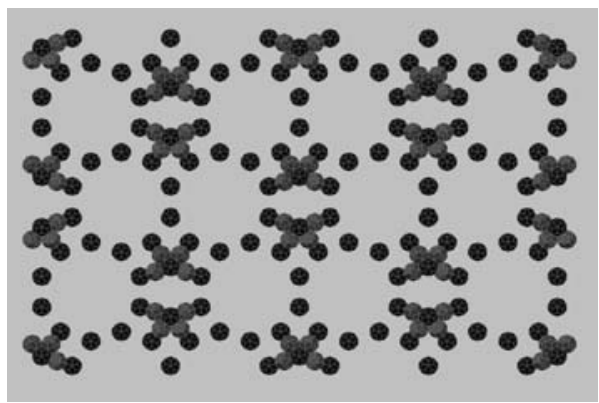

Fig. 13. 2 differently shaped 5D elements with vertical symmetry planes

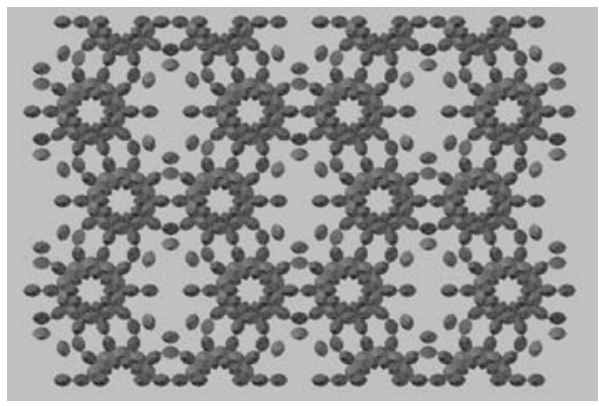

Fig. 15. 3 differently shaped 4D elements with vertical symmetry planes

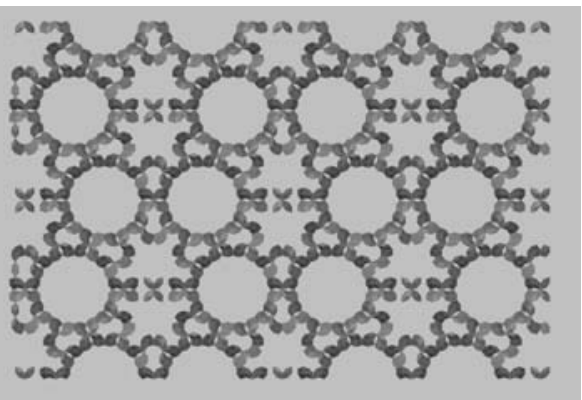

Fig. 17. 4 differently shaped 3D elements without symmetry planes 
Moved intersection planes cut series of periodical plane-tiling patterns from the mosaics. These sections transform into each other in infinitely repeatable sequences of frames of animations. Examples constructed from 3-models of 3-12 dimensional cubes can be seen on the homepage [7]. Fig. 18 shows a stochastically chosen horizontal intersection of the second mosaic.

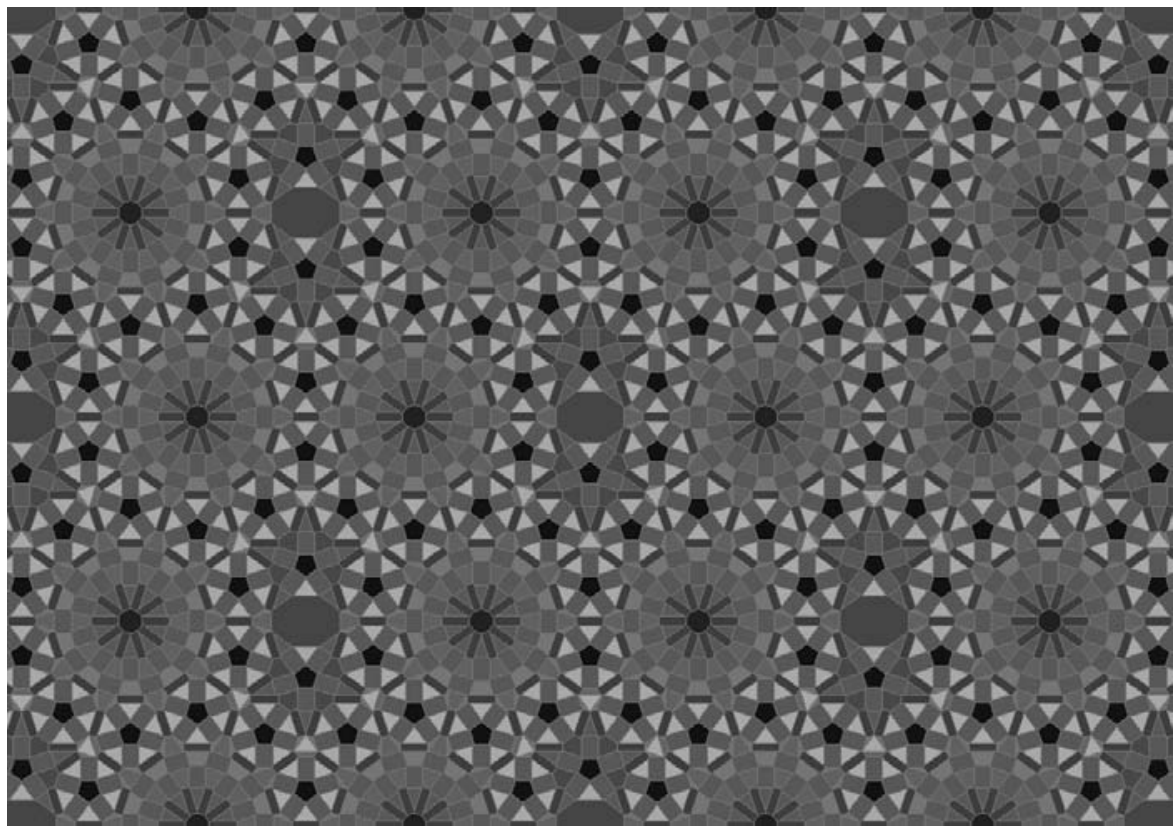

Fig. 18. Stochastically chosen horizontal intersection of the second mosaic

\section{Systematization of available and new results}

Table IV shows the systematization of former results of the Author, regarding the different ways of the modeling of more-dimensional cubes by the above described method for constructions of space-filling periodical mosaics and plane-tiling patterns gained from these. The results are signed with square brackets according to the reference list. References in round brackets mean that the instances are only mentioned without figures. The starred items have animated planar tessellation series as well, gained from the given spatial mosaics. Most of them can be seen in [7].

Modification means that the angles among the initial edges and/or their length are different but the above described symmetry properties are kept to the largest extent possible. The formula $l-1=k$ means that the model of the $k$-cube is constructed with $k+1=l$ initial edges arranged in rotational symmetry but leaving an edge out. It is possible to apply this method with more edges left out $(k+o=l)$ as well. 
Table IV

Systematization of available and new results

\begin{tabular}{|c|c|c|c|c|c|c|c|c|c|}
\hline $\begin{array}{l}\text { Dimension/ } \\
\text { Method }\end{array}$ & 4 & 5 & 6 & 7 & 8 & 9 & 10 & 11 & 12 \\
\hline $\begin{array}{l}\text { Without } \\
\text { modification }\end{array}$ & [11] & $\begin{array}{l}{[2],} \\
{[11]^{*}}\end{array}$ & $\begin{array}{l}{[8],} \\
{[11]^{*}}\end{array}$ & [2] & {$[9]^{*}$} & {$[10]^{*}$} & $*$ & & $\begin{array}{l}{[2],} \\
{[11]^{*}}\end{array}$ \\
\hline $\begin{array}{l}\text { With each } \\
\text { derived } \\
i \text {-cube }\end{array}$ & {$[11]^{*}$} & {$[11]^{*}$} & {$[11]^{*}$} & 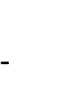 & - & & - & & \\
\hline $\begin{array}{l}\text { With } \\
\text { modification }\end{array}$ & [11] & {$[9]$} & [2], [3] & {$[10]$} & - & $\begin{array}{l}{[2]^{*},} \\
([9])\end{array}$ & {$[1]^{*}$} & & - \\
\hline$l-1=k$ & - & {$[2]$} & $([2])$ & {$[11]$} & - & - & - & & - \\
\hline$(l-o)=k$ & - & - & {$[2],[11]$} & & - & [2] & & & \\
\hline
\end{tabular}

All available solutions can be restructured in fractals as it is described in some papers of the author, for instance in [2], [8], [11]. Modified or specially chosen initial edges of the 3-model of the more-dimensional cubes give several possibilities to construct spatial tessellations. An example for this is described in [12].

\section{Concluding remarks}

Recent paper deals with the two new results of periodical tessellations based on the above described special 3-model of the 10-cube. These have horizontally symmetric layers of the spatial mosaic elements. The arrangements of these stones follow the rotational symmetry of the 10 -cube. Thus the repeated honeycombs of the spatial tessellations are structured as right prisms. The faces of the prisms are symmetry planes of the models of derived $3<j<10$ dimensional elements, bordering the honeycombs. The described results are probably the simplest solutions of this kind of arrangements. Examples of other tessellations ground the conjecture that also these space-filling mosaics could be built with more elements as well, following the same geometrical structure.

Recent results make possible the construction of periodical space-filling mosaics based on the modified 3-model of the 11-cube. Ten initial edges have the same arrangement like in the above model of the 10-cube. Simple and coherent solutions can be given if the 11th edge is vertical and the upper endpoint of this is in the horizontal plane of the upper endpoints of the other 10 edges spread of rotationally symmetric around the 11th one.

The creation of the constructions and figures for this paper was aided by the AutoCAD program and AutoLisp routines; latter is developed by the Author.

\section{Acknowledgement}

The present scientific contribution is dedicated to the $650^{\text {th }}$ anniversary of the foundation of the medieval university of Pécs, Hungary. 


\section{References}

[1] Vörös L. N-zonotopes and their images, from hypercube to art in geometry, Proceedings of Library of Congress Cataloguing-in-Publication Data, W. Gunter (Editor on chief) and ISGG, Proceedings of the 13th International Conference on Geometry and Graphics, Dresden, Germany, 4-8 August 2008 (electronic book/ISGG, G. Weiss (Ed), Dresden: ERZSCHLAG GbR, Aue, 2008, http://geometria.mik.pte.hu (last visited 1 February 2015).

[2] Vörös L. Sets of space-filling zonotopes with connection to art and design, ProceedingsDVD of the 14th International Conference on Geometry and Graphics, 5-9 August 2010, Kyoto, Japan, International Society for Geometry and Graphics, Article No. 231, 2010, http://geometria.mik.pte.hu (last visited 1 February 2015).

[3] Vörös L. Reguläre Körper und mehrdimensionale Würfel, KoG Scientific and Professional Journal of the Croatian Society for Geometry and Graphics, No. 9, 2005, pp. 21-27, http://master.grad.hr/hdgg/kog_stranica/kog9.pdf (last visited 1 February 2015).

[4] http://en.wikipedia.org/wiki/Hypercube (last visited 1 February 2015).

[5] Coxeter H. S. M. Regular polytopes, 2nd ed., The MacMillan Company, New York, 1963.

[6] Towle R. Zonotopes, symmetrical-structures, 2008, http://zonotopia.blogspot.com (last visited 1 February 2015).

[7] http://geometria.mik.pte.hu (last visited 1 February 2015).

[8] Vörös L. Two- and three-dimensional tilings based on a model of the six-dimensional cube, KoG Scientific and Professional Journal of the Croatian Society for Geometry and Graphics, No. 10, 2006, pp. 19-25, http://master.grad.hr/hdgg/kog_stranica/kog10.pdf (last visited 1 February 2015).

[9] Vörös L. Two- and three-dimensional tiling on the base of higher-dimensional cube mosaics, Proceedings of the 7th International Conference on Applied Informatics, Eger, Hungary, 28 January - 2 February 2007, Vol. 1, 2007, pp. 185-192, http://geometria. mik.pte.hu (last visited 1 February 2015).

[10] Vörös L. Space-filling mosaics and (animated) plane-tiling patterns, Symmetry, Culture and Science, Vol. 20, No. 1-4, 2009, pp. 297-308, http://geometria.mik.pte.hu (last visited 1 February 2015).

[11] Vörös L. News on sets of space-filling zonotopes, Aplimat, Journal of Applied Mathematics, Vol. 5, No. 1, 2012, pp. 247-256.

[12] Vörös L. Space-filling mosaics of 3D models of 3-13D cubes related to regular and semiregular polyhedra, Proceedings of the 4th International Scientific Conference on Geometry and Graphics moNGeometrija, Vlasina, Serbia, 20-22 June 2014, Vol. 2, 2014, pp. 22-31, http://mongeometrija.com/attachments/article/377/Contents_Vol 2.pdf, (last visited 1 February 2015). 